\title{
The primordial end calculus of prime numbers and mathematics
}

\author{
Vinoo Cameron \\ Hope research, Athens, Wisconsin, USA \\ E-mail:9900@frontier.com
}

\begin{abstract}
This Manuscript on the end primordial calculus of mathematics is a new discovery of the spiral nature of the entire mathematical grid at 1:3 by the precise and absolute concordance of regular number spirals and the Prime number spirals based on numbers and their spaces by grid. It is exclusive to IJAMR which has published 8 papers of the author on this new mathematics. The manuscript has NOT been offered to any other journal in the world .The editorial board of Princeton University, USA, Annals of mathematics had been duly informed by letter of the new discovery of the concordance of prime numbers spirals with regular number spirals, but for the sake of fidelity.

Mathematics is not complexity, but simplicity, the configuration of 1 is spiral .The relationship between pure mathematical numbers and empty space is a primordial relationship, and well defined by gaps, plus it has been validated by the author by the Publishing of the pure continuous Den-Otter Prime number sieve at 1/6 and 5/6 ( and $1 / 3$ and $2 / 3$ ), and these prime sieves are reversible. Thus the relationship of the configuration of 1 is in two planes that expand in the frame of (5/6 and 1/6) and (1/3 and 2/3)are represented by spiral configuration, expressed by these numbers, as in :All prime numbers spirals are assigned infinitely by the simple -1 offset of the two spiral numbers cords
\end{abstract}

$1 / 3+2 / 3=1$

$5 / 6+1 / 6=1$

$1 / 3-1 / 6=1 / 6$

$5 / 6-2 / 3=1 / 6$

$1 / 3+1 / 6=0.5$

$5 / 6+2 / 3=1.5$

$1.5 / 0.5=3$

Note: the above is also confirmed by Arabian numerical shown below.

Primordial mathematics as created by the creator is absolute and precise whilst the mathematics invented by man is approximate .Current Mathematics of a thousand years has accomplished much by approximate theory, and it is very possible to land a man on the moon by approximate mathematics, but to precisely understand mass and energy, the nucleus of mass and the vastness of space, one must understand the precise nature and curvature of space.

Space and mathematics are curved precisely at 1:3 from primordial times( the basis for the $\pi$ as shown under the publication Unified Theorem), and the warping of space is related to the 1:3 separation at precise 19 correct degrees separation and the precise right angled separation at 1:3 is at 360/19 degrees. This mathematics is about the curved lay of the grid of mathematics and cannot be understood by the current linear obsession of mathematics, but the readers are immediately referred to the 8 published papers of the author as referenced below. The calculus below virtually negates the current mathematics ideas of linear ascension theories of the past 500 years. This calculus is true for all prime numbers. This involves a precise "combing" of all prime number spirals around a half line. This is clearly and singularly the solution to prime number spiral calculus, which is fairly simple after this discovery. The ruminations of George Riemann and others of the past 500 years in linear ascension mathematics of prime numbers are non- functional irrelevant mathematics, just collateral observations of the correct mathematics which is presented here as "Primum movens" of mathematics. In Mathematics there is only a single best resolution, whilst collateral equations abound, as they have for 500 years for prime numbers which we have proved are basically by two chords and ascend by a half-line. Einstein's observations and ruminations likewise are collateral and not central to the fact that the universe is precisely curved, and ascension of prime numbers are by two spiral chords. 


\section{Introduction}

This massive mathematics can only be introduced by the published references provided below. Basically the entire mathematics is at 1:3 at 19 correct degrees by spatial configuration which is shown under. The basic novel continuous Prime number sieve at $1: 3$, at $(5 / 6: 1 / 6)$ and $(2 / 3: 1 / 3)$ has been recently published, yet unnoticed. It is the Den- Otter prime sieve and is reversible

The word "Spiral" for the purposes of this paper indicates the three numbers of concordant gaps around a prime number. The symbol (n_) represents a gap between numbers as an example for the prime number 11 the following are the spiral gaps.

By regular numbers at prime number 11: 9_11_13

By prime numbers at prime number 11: 5_11_17

The Basic Mathematics of concordance of spiral numbers is at gaps 12 and gap 4 as in the example above $(9+11+13)=(5+11+17)=33$ for regular numbers and is the base at $(12 / 4=3)$ and the fact that at this grid alone the total additional values for the regular spiral numbers at 4 span equal those of the Prime number spirals at 12 span, as shown further below, and is a constant, so is the constant 32 .The mentioned Spiral constants are constant throughout the entire mathematical grid between the Prime number spirals and the regular prime number spirals as follows, at the half line( Spiral gap 10,12,14,16,18,20,22,24, 26, 28,30. ...and so on.

\subsection{Spiral progression of numbers/prime numbers by gap value}

Constant 4 and 12 (example for prime numbers 19, 41, 43...)

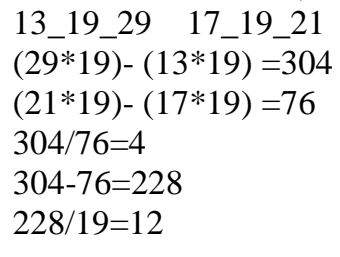

31_41_47 39_41_43

$(47 * 41)-(41 * 31)=656$

$(43 * 41)-(39 * 41)=164$

$656 / 164=4$

$656-164=492$

$492 / 41=12$

37_43_53 41_43*45

$(53 * 43)-(43 * 37)=688$

$(45 * 43)-43 * 41)=172$

$688 / 172=4$

$688-172=516$

$516 / 43=12$

67_73_83 71_73_75

$(83 * 73)-(73 * 71)=1168$

$(75 * 73)-(73 * 71=292$

$1168 / 292=4$

$1168-292=876$

$876 / 73=12$

Gap 18 spirals: Constant 4.5 and $14($ example prime numbers $29,31,47$ )

17_29_37 27_29_31

$(37 * 29)-(29 * 19)=522$

$((29 * 31)-(29 * 27)=116$

$522 / 16=4.5$

$522-116=406$

$406 / 29=14$

23_31_41 29_31_33

$(41 * 31)-(31 * 23)=558$ 
$(33 * 31-(29 * 31)=124$

$558 / 124=4.5$

$558-124=434$

$434 / 31=14$

41_47_59 45_47_49

$(59 * 47)-(47 * 41)=846$

$(49 * 47)-(47 * 45)=188$

$846 / 188=4.5$

$846-188=658$

$658 / 47=14$

Gap Spiral 20: Constant 5 and 16 (example Prime numbers 59, 97, 139, 151)

47_59_67 57_59_61

$(67 * 59)-(59 * 47)=1180$

$(59 * 61)-(59 * 57)=236$

$1180 / 236=5$

$1180-236=944$

$944 / 59=16$

83_97_103 95_97_99

$(103 * 97)-(97 * 83)=1940$

$(99 * 97)-(95 * 97=388$

$1940 / 388=5$

$1940-388=1552$

$1552 / 97=16$

131_139_151 95_97_99

$(151 * 139)-(139 * 131)=2780$

$(141 * 139)-(139 * 137)=556$

$2780 / 556=5$

$2780-556=2224$

$2224 / 139=16$

\subsection{Indirect assignment of all spiral values of prime numbers by the offset of prime number} dual chains at $\mathbf{- 1}$

Please note the -1base offset arrangement that delineate the spiral values for each prime number. Precise values are obtained from the new continuous Prime number sieve at 5/6, 1/6 and 1/3, 2/3(Den-Otter prime number Sieve recently published by the author).The spiral gap is indicated as Prime number (spiral span)

\begin{tabular}{ccc} 
& Spiral gap & \\
\hline 1 & $5(10)$ & 11 \\
3 & $7(10)$ & 13 \\
5 & $11(12)$ & 17 \\
7 & $13(12)$ & 19 \\
11 & $17(12)$ & 23 \\
13 & $19(16)$ & 29 \\
17 & $23(14)$ & 31 \\
19 & $29(18)$ & 37 \\
23 & $31(18)$ & 41 \\
29 & $37(14)$ & 43 \\
31 & $41(16)$ & 47 \\
37 & $43(16)$ & 53 \\
41 & $47(18)$ & 59 \\
43 & $53(18)$ & 61 \\
47 & $59(20)$ & 67 \\
53 & $61(18)$ & 71 \\
59 & $67(14)$ & 73 \\
61 & $71(18)$ & 79
\end{tabular}




$\begin{array}{lll}67 & 73(16) & 83 \\ 71 & 79(18) & 89 \\ 73 & 83(24) & 97 \\ 79 & 89(22) & 101 \\ 83 & 97(20) & 103 \\ 89 & 101(18) & 107 \\ 97 & 103(12) & 109 \\ 101 & 107(12) & 113 \\ 103 & 109(24) & 127 \\ 107 & 113(24) & 131 \\ 109 & 127(28) & 137 \\ 113 & 131(26) & 139 \\ 127 & 137(22) & 149 \\ 131 & 139(20) & 151 \\ 137 & 149(20) & 157 \\ 139 & 151(24) & 163 \\ 149 & 157(18) & 167 \\ 151 & 163(22) & 173 \\ 157 & 167(22) & 179 \\ 163 & 173(18) & 181 \\ 167 & 179(24 & 191 \\ 173 & 181(20) & 193 \\ 179 & 191(18) & 197 \\ 181 & 193(18) & 199 \\ 191 & 197(20) & 211 \\ 193 & 199(30) & 223 \\ 197 & 211(30) & 227 \\ 199 & 223(30) & 229 \\ 211 & 227(22) & 233 \\ 223 & 229(16) & 239 \\ 227 & 233(14) & 241 \\ 229 & 239(22) & 251 \\ 233 & 241(24) & 257 \\ 239 & 251(24) & 263 \\ 241 & 257(28) & 269 \\ 251 & 263(20) & 271 \\ 257 & 269(20) & 277 \\ 263 & 271(18) & 281 \\ 269 & 277(14) & 283 \\ 271 & 281(22) & 293 \\ 277 & 283(36) & 307 \\ 281 & 293(34) & 311 \\ 283 & 307(30) & 313 \\ 293 & 311(24) & 317 \\ 307 & 313(24) & 331 \\ 311 & 317(26) & 337 \\ 313 & 331(34) & 347 \\ 317 & 337(32) & 349 \\ 331 & 347(22) & 353 \\ 337 & 349(22) & 359 \\ 347 & 353(20) & 367 \\ 349 & 359(24) & 373 \\ 353 & 367(26) & 379 \\ 359 & 373(24) & 383 \\ 367 & 379(22) & 389 \\ 373 & 383(24) & 397 \\ 379 & 389(22) & 401 \\ 383 & 397(26) & 409 \\ 389 & 401(30) & 419 \\ 397 & 409(24) & 421 \\ & & \\ 93 & & \\ 139 & \end{array}$




\begin{tabular}{|c|c|c|}
\hline 401 & 419 (30) & 431 \\
\hline 409 & $421(24)$ & 433 \\
\hline 419 & $431(20)$ & 439 \\
\hline 421 & 433 (22) & 443 \\
\hline 431 & 439 (18) & 449 \\
\hline 433 & 443 (24) & 457 \\
\hline 439 & 449 (22) & 461 \\
\hline 443 & 457 (20) & 463 \\
\hline 449 & $461(18)$ & 467 \\
\hline 457 & 463 (22) & 479 \\
\hline 461 & 467 (26) & 487 \\
\hline 463 & 479 (28) & 491 \\
\hline 467 & 487 (32) & 499 \\
\hline 479 & 491 (24) & 503 \\
\hline 487 & 499 (22) & 509 \\
\hline 491 & $503(30)$ & 521 \\
\hline 499 & $509(24)$ & 523 \\
\hline 503 & $521(38)$ & 541 \\
\hline 509 & $523(38)$ & 547 \\
\hline 521 & $541(36)$ & 557 \\
\hline 523 & $547(40)$ & 563 \\
\hline 541 & 557 (29) & 569 \\
\hline 547 & $563(24)$ & 571 \\
\hline 557 & $569(20)$ & 577 \\
\hline 563 & $571(24)$ & 587 \\
\hline 569 & 577 (24) & 593 \\
\hline 571 & 587 (28) & 599 \\
\hline 577 & 593 (24) & 601 \\
\hline 587 & $599(20)$ & 607 \\
\hline 593 & $601(20)$ & 613 \\
\hline 599 & 607 (18) & 617 \\
\hline 601 & 613 (18) & 619 \\
\hline 607 & 617 (24) & 631 \\
\hline 613 & 619 (28) & 641 \\
\hline 617 & $631(26)$ & 643 \\
\hline 619 & $641(28)$ & 647 \\
\hline 631 & 643 (22) & 653 \\
\hline 641 & 647 (18) & 659 \\
\hline 643 & 653 (18) & 661 \\
\hline 647 & 659 (26) & 673 \\
\hline 653 & $661(24)$ & 677 \\
\hline 659 & 673 (24) & 683 \\
\hline 661 & 677 (30) & 691 \\
\hline 673 & 683 (28) & 701 \\
\hline 677 & $691(32)$ & 709 \\
\hline 683 & 701 (36) & 719 \\
\hline 691 & 709 (36) & 727 \\
\hline 701 & 719 (32) & 733 \\
\hline 709 & 727 (30) & 739 \\
\hline 719 & 733 (24) & 743 \\
\hline 727 & 739 (24) & 751 \\
\hline 733 & 743 (24) & 757 \\
\hline 739 & 751 (22) & 761 \\
\hline 743 & 757 (26) & 769 \\
\hline 751 & 761 (22) & 773 \\
\hline 757 & 769 (30) & 787 \\
\hline 761 & 773 (36) & 797 \\
\hline 769 & 787 (40) & 809 \\
\hline 773 & 797 (38) & 811 \\
\hline
\end{tabular}




\begin{tabular}{cll}
787 & $809(34)$ & 821 \\
797 & $811(26)$ & 823 \\
809 & $821(18)$ & 827 \\
811 & $823(18)$ & 829 \\
821 & $827(18)$ & 839 \\
823 & $829(20)$ & 853 \\
827 & $839(30)$ & 857 \\
829 & $853(30)$ & 859 \\
839 & $857(24)$ & 863 \\
853 & $859(24)$ & 877 \\
857 & $863(24)$ & 881 \\
859 & $877(24)$ & 883 \\
So on & & \\
\hline
\end{tabular}

And so on for all prime number spirals, and a clear calculus to predict all prime number spiral values, based on this end calculus and what follows below. Most clearly the spirals are predictable and constant.

\subsection{Schematic distribution of the spirals by the half-line and the two spirals (the actual spirals are at a tangent to the half-line)}

\begin{tabular}{|c|c|c|c|c|c|c|c|c|c|c|c|c|c|c|c|c|}
\hline 24 & 22 & 20 & 18 & 16 & 14 & 12 & 10 & 1 & 10 & 12 & 14 & 16 & 18 & 20 & 22 & 24 \\
\hline & & & & & & & & 2 & & & & & & & & \\
\hline & & & & & & & & 3 & & & & & & & & \\
\hline & & & & & & & & 4 & & & & & & & & \\
\hline & & & & & & & & 5 & & & & & & & & \\
\hline & & & & & & & & 6 & & & & & & & & \\
\hline & & & & & & & & 7 & 7 & & & & & & & \\
\hline & & & & & & & & 8 & & & & & & & & \\
\hline & & & & & & & & 9 & & & & & & & & \\
\hline & & & & & & & & 10 & & & & & & & & \\
\hline & & & & & & 11 & & 11 & & 11 & & & & & & \\
\hline & & & & & & & & 12 & & & & & & & & \\
\hline & & & & & & 13 & & 13 & & & & & & & & \\
\hline & & & & & & & & 14 & & & & & & & & \\
\hline & & & & & & & & 15 & & & & & & & & \\
\hline & & & & & & & & 16 & & & & & & & & \\
\hline & & & & & & 17 & & 17 & & 17 & & & & & & \\
\hline & & & & & & & & 18 & & & & & & & & \\
\hline & & & & & & & & 19 & & & & 19 & & & & \\
\hline & & & & & & & & 20 & & & & & & & & \\
\hline & & & & & & & & 21 & & & & & & & & \\
\hline & & & & & & & & 22 & & & & & & & & \\
\hline & & & & & 23 & & & 23 & & & 23 & & & & & \\
\hline & & & & & & & & 24 & & & & & & & & \\
\hline & & & & & & & & 25 & & & & & & & & \\
\hline & & & & & & & & 26 & & & & & & & & \\
\hline & & & & & & & & 27 & & & & & & & & \\
\hline & & & & & & & & 28 & & & & & & & & \\
\hline & & & 29 & & & & & 29 & & & & & 29 & & & \\
\hline & & & & & & & & 30 & & & & & & & & \\
\hline & & & 31 & & & & & 31 & & & & & 31 & & & \\
\hline & & & & & & & & 32 & & & & & & & & \\
\hline & & & & & & & & 33 & & & & & & & & \\
\hline & & & & & & & & 34 & & & & & & & & \\
\hline & & & & & & & & 35 & & & & & & & & \\
\hline & & & & & & & & 36 & & & & & & & & \\
\hline & & & & & 37 & & & 37 & & & 37 & & & & & \\
\hline & & & & & & & & 38 & & & & & & & & \\
\hline & & & & & & & & 39 & & & & & & & & \\
\hline & & & & & & & & 40 & & & & & & & & \\
\hline & & & & 41 & & & & 41 & & & & 41 & & & & \\
\hline & & & & & & & & 42 & & & & & & & & \\
\hline & & & & 43 & & & & 43 & & & & 43 & & & & \\
\hline & & & & & & & & 44 & & & & & & & & \\
\hline & & & & & & & & 45 & & & & & & & & \\
\hline & & & & & & & & 46 & & & & & & & & \\
\hline & & & 47 & & & & & 47 & & & & & 47 & & & \\
\hline & & & & & & & & 48 & & & & & & & & \\
\hline
\end{tabular}




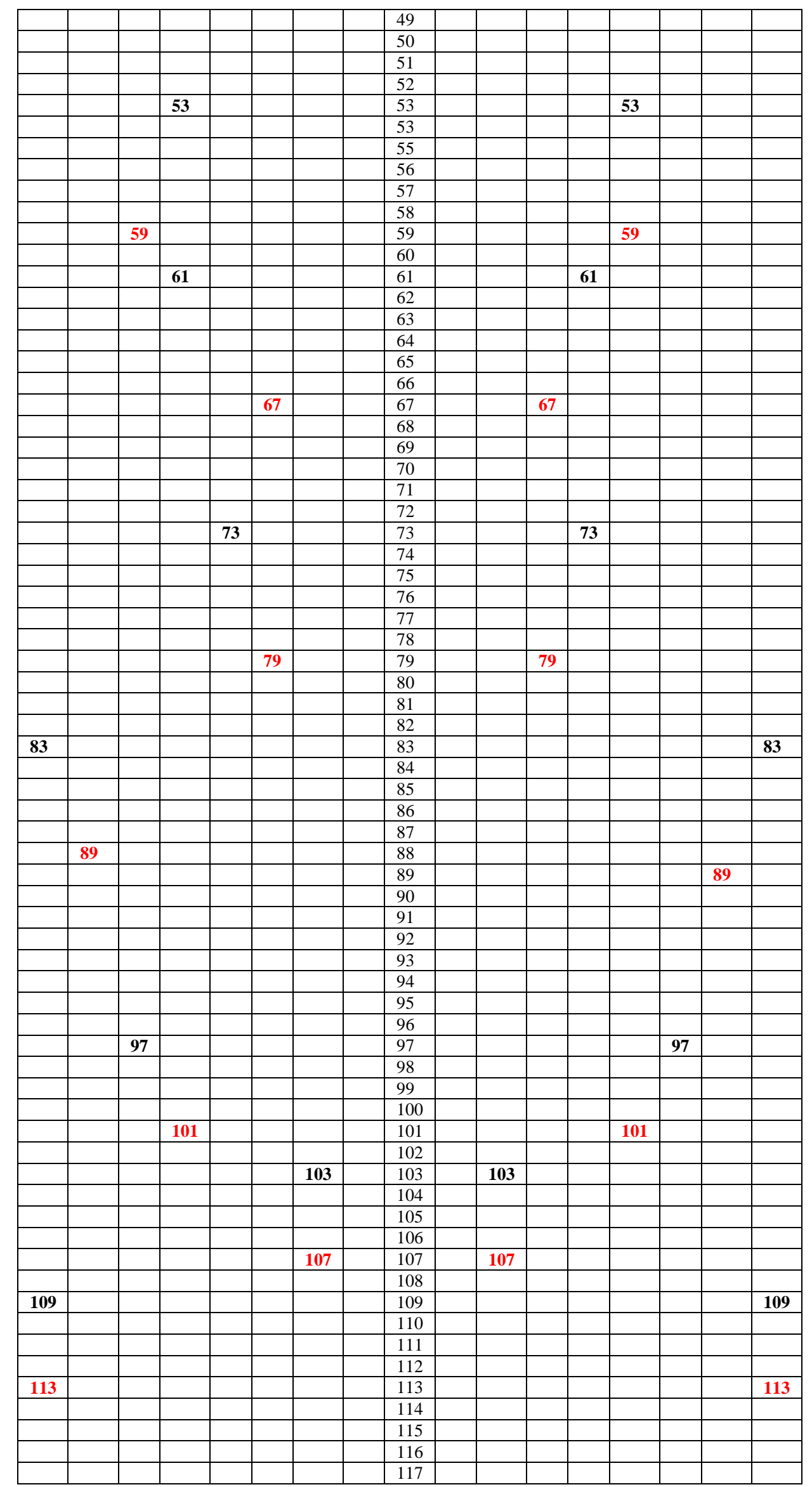




\begin{tabular}{|l|l|l|l|l|l|l|l|l|l|l|l|l|l|l|l|l|}
\hline & & & & & & & & 119 & & & & & & & & \\
\hline & & & & & & & & 120 & & & & & & & & \\
\hline & & & & & & & & 121 & & & & & & & & \\
\hline & & & & & & & & 123 & & & & & & & & \\
\hline & & & & & & & & 124 & & & & & & & & \\
\hline & & & & & & & & 125 & & & & & & & & \\
\hline & & & & & & & & 126 & & & & & & & & \\
\hline & $\mathbf{1 2 7}$ & & & & & & & 127 & & & & & & & $\mathbf{1 2 7}$ & \\
\hline 24 & 22 & 20 & 18 & 16 & 14 & 12 & 10 & 127 & 10 & 12 & 14 & 16 & 18 & 20 & 22 & 24 \\
\hline & & & & & & & & & & & & & & & & \\
\hline
\end{tabular}

Unscrambled distribution table of the spiral values: These values are for prime numbers and the concordant spirals of regular numbers at fixed span of 4 (absolute concordance is at 1:3 values as shown below): Prime number is in red, the collateral prime number on the left. On the right are the regular number spiral coordinates of the same prime numbers:

\begin{tabular}{|c|c|c|c|c|c|}
\hline $10 \mathrm{~S}$ & Prime no & & & Prime no & \\
\hline 1 & 5 & 11 & 3 & 5 & 7 \\
\hline 3 & 7 & 11 & 5 & 7 & 9 \\
\hline \multicolumn{6}{|l|}{$12 \mathrm{~S}$} \\
\hline 5 & 11 & 17 & 9 & 11 & 13 \\
\hline 7 & 13 & 19 & 11 & 13 & 15 \\
\hline 11 & 17 & 23 & 15 & 17 & 19 \\
\hline 97 & 103 & 109 & 101 & 103 & 105 \\
\hline 101 & 107 & 113 & 105 & 107 & 109 \\
\hline 1481 & 1487 & 1493 & 1485 & 1487 & 1489 \\
\hline \multicolumn{6}{|l|}{$14 \mathrm{~S}$} \\
\hline 17 & 23 & 31 & 21 & 23 & 25 \\
\hline 29 & 37 & 43 & 35 & 37 & 43 \\
\hline 59 & 67 & 73 & 65 & 67 & 69 \\
\hline 227 & 233 & 241 & 231 & 233 & 235 \\
\hline 269 & 277 & 283 & 275 & 277 & 279 \\
\hline 1277 & 1283 & 1291 & 1281 & 1283 & 1285 \\
\hline 1289 & 1297 & 1303 & 1295 & 1297 & 1299 \\
\hline \multicolumn{6}{|l|}{$16 \mathrm{~S}$} \\
\hline 13 & 19 & 29 & 17 & 19 & 21 \\
\hline 31 & 41 & 47 & 39 & 41 & 43 \\
\hline 37 & 43 & 53 & 41 & 43 & 45 \\
\hline 67 & 73 & 83 & 71 & 73 & 75 \\
\hline 223 & 229 & 239 & 227 & 229 & 231 \\
\hline 1087 & 1093 & 1103 & 1091 & 1093 & 1095 \\
\hline 1423 & 1429 & 1439 & 1427 & 1429 & 1431 \\
\hline 1471 & 1481 & 1487 & 1479 & 1481 & 1483 \\
\hline \multicolumn{6}{|l|}{$18 \mathrm{~S}$} \\
\hline 17 & 29 & 37 & 27 & 29 & 31 \\
\hline 23 & 31 & 41 & 29 & 31 & 33 \\
\hline 41 & 47 & 59 & 45 & 47 & 49 \\
\hline 43 & 53 & 61 & 51 & 53 & 55 \\
\hline 53 & 61 & 71 & 59 & 61 & 63 \\
\hline 61 & 71 & 79 & 69 & 71 & 73 \\
\hline 71 & 79 & 89 & 77 & 79 & 81 \\
\hline 89 & 101 & 107 & 99 & 101 & 103 \\
\hline 149 & 157 & 167 & 155 & 157 & 167 \\
\hline 163 & 173 & 181 & 171 & 173 & 175 \\
\hline 179 & 191 & 197 & 189 & 191 & 197 \\
\hline 181 & 193 & 199 & 191 & 193 & 195 \\
\hline 263 & 271 & 281 & 269 & 271 & 273 \\
\hline 431 & 439 & 449 & 437 & 439 & 441 \\
\hline 449 & 461 & 467 & 459 & 461 & 463 \\
\hline 599 & 607 & 613 & 605 & 607 & 609 \\
\hline 601 & 613 & 619 & 611 & 613 & 615 \\
\hline 641 & 647 & 659 & 645 & 647 & 649 \\
\hline
\end{tabular}




\begin{tabular}{|c|c|c|c|c|c|}
\hline 643 & 653 & 661 & 651 & 653 & 655 \\
\hline 809 & 821 & 827 & 819 & 821 & 823 \\
\hline 811 & 823 & 829 & 821 & 823 & 825 \\
\hline 821 & 827 & 839 & 825 & 827 & 829 \\
\hline 1091 & 1097 & 1109 & 1095 & 1097 & 1099 \\
\hline 1213 & 1223 & 1231 & 1221 & 1223 & 1225 \\
\hline 1279 & 1289 & 1287 & 1287 & 1289 & 1291 \\
\hline 1283 & 1291 & 1301 & 1289 & 1291 & 1293 \\
\hline 1471 & 1483 & 1489 & 1481 & 1483 & 1485 \\
\hline \multicolumn{6}{|l|}{$20 \mathrm{~S}$} \\
\hline 47 & 59 & 67 & 57 & 59 & 61 \\
\hline 83 & 97 & 103 & 95 & 97 & 99 \\
\hline 131 & 139 & 151 & 137 & 139 & 141 \\
\hline 137 & 149 & 157 & $147 *$ & 149 & 151 \\
\hline 153 & 163 & 173 & 161 & 163 & 165 \\
\hline 191 & 197 & 211 & $195^{*}$ & 197 & 199 \\
\hline 257 & 269 & 277 & 267 & 269 & 271 \\
\hline 369 & 379 & 389 & 377 & 379 & 381 \\
\hline 443 & 457 & 463 & 455 & 457 & 459 \\
\hline 557 & 567 & 577 & 565 & 567 & 569 \\
\hline 587 & 599 & 607 & 597 & 599 & 601 \\
\hline 593 & 601 & 613 & 599 & 601 & 603 \\
\hline 1013 & 1021 & 1033 & 1019 & 1021 & 1023 \\
\hline 1019 & 1031 & 1039 & 1029 & 1031 & 1033 \\
\hline 1031 & 1039 & 1051 & 1037 & 1039 & 1041 \\
\hline 1049 & 1061 & 1069 & 1059 & 1061 & 1063 \\
\hline 1217 & 1229 & 1237 & 1227 & 1229 & 1231 \\
\hline
\end{tabular}

\subsection{End calculus of mathematics, by Arabic numerals 0-3^2 (1:3), the correct numbers theorem}

We demonstrate the concordance of regular spiral numbers $(+2)$ and the spiral prime numbers of the Spiral gap 16, 14, 18 , and 20 to show concordance in the spirals. This is a very major proof against current mathematics number and prime number theory and their approach to "random" Prime numbers, which is non-existent, as there are no random prime numbers which has been a vain imagination of current mathematics throughout the centuries.

$0,1,2,3,4,5,6$

The above rational numbers are arranged so that 3 are at the half line of 0 and 6 . The exclusive value of 3 as a number is represented by

$3 * 2=6$ and $6 / 6=1(1: 3)$

$3 * 2=6$ and $3 * 4=12 ; 12 / 6=0.5(0.5)$

$0,(1) 2=0 / 2=0$

$0,1,(2), 3,4=(2 * 1 / 4=0.5)$

$0,1,2,(3), 4,5,6=(3 * 2 / 6=1)$

$0,1,2,3,(4), 5,6,7,8=(4 * 3 / 8=1.5)$

$0,1,2,3,4,(5), 6,7,8,9,10=(5 * 4 / 10=2)$

$0,1,2,3,4,5,(6), 7,8,9,10,11,12=(6 * 5 / 12=2.5)$

$0,1,2,3,4,5,6,(7), 9,10,11,12,13,14=(7 * 6 / 14=3)$

$0,1,2,3,4,5,6,7,(8), 9,10,11,12,13,14,15,16=(8 * 7 / 16=3.5)$

$0,1,2,3,4,5,6,7,8,(9), 10,11,12,13,14,15,16,17,18,(72 / 18=4)$

\section{Conclusion:}

$1: 4$ span is proportional to $1: 3\left[3\right.$ and $\left.3^{\wedge} 2(9)\right]$ [ 3 and $3^{*} 2(6)$ ] by a factor of $6\left(3^{\wedge} 2-3\right)$ and $3(4-1)$, and $0.5 / 60(1 / 360 / 3)$ is the logical offset at the base of mathematics as published separately. Thus clearly the span of mathematics by numbers and prime numbers is divergent at 3 and convergent at 6 , in concordance to $1: 4$

Note at 19 , value is 9 :

At 3 values is 1 
At 5 the value is 2

At 7 values are 3

At 9 value is $4\left(2^{\wedge} 2\right)$

At 19 value is $9\left(3^{\wedge} 2\right)$

$(19-7) /(9-5)=3$

$(9-3) /(4-2)=3$

$0,1,2,3,4,5,6,7,8,9,10,11,12,13,14,15,16,17,18,(19), 20,21,22,23,24,25,26,27,28,29,30,31,31,32,33,34,35,26,37,38$

$(19 * 18) / 38=9\left(3^{\wedge} 2\right)$

So 19 as a proportionate prime number does represent 1:3 as we have said, but we have had to present mathematics in the most rudimentary manner, to make many mathematicians understand the primordial arrangement.

\subsection{The configuration of 1 at (5/6and 1/6) and (1/3 and 2/3) the author has prime number sieve at these proportions (den otter sieve)}

This is the basic example of the precise configuration of mathematics and the understanding applies to the whole grid of the whole mathematics, and that's the way mathematics is, AND when the space numerations of the sets of calculation are applied to the ascension of mathematics, these are precise and very concordant to the grid of mathematics as shown under, but the understanding is difficult, but that is the lay of mathematics, it is not linear, it is curved precisely by prime numbers that are curved in the spatial expanse of mathematics. The author cannot express it any better, and the current understanding of mathematics is quite confused.

This diagram is precisely representative of the concordance of prime numbers and coned expansion of mathematics of which prime numbers are integral. The trigonometric association is a separate mathematics. This below is the general understanding of prime numbers and their relationship to curved space. Any mathematician, who still thinks that the numbers 2 and 3 are in the prime number configuration, should depart from this discussion and never come back, till they have rediscovered mathematical logic. Mathematically by serial numbers this is totally predictable, since the configuration is 4 alternating with 2 , a no- brainer.

Prime number/ Pseudo prime number $/ 6=1 / 6$ or $5 / 6$, thus:

$1 / 6+5 / 6=1$

$5 / 6+7 / 6=2$

$7 / 6+11 / 6=3$

$11 / 6+13 / 6=4$

$13 / 6+17 / 6=5$

$17 / 6+19 / 6=6$

$19 / 6+23 / 6=7$

$[((19 / 6) / 7) /((23 / 6) / 7)=1]$

$23 / 6+25 / 6=8$

$25 / 6+29 / 6=9$

$29 / 6+31 / 6=10$

$31 / 6+35 / 6=11$

$35 / 6+37 / 6=12$

$37 / 6+41 / 6=13$

$41 / 6+43 / 6=14$

$43 / 6+47 / 6=15$

$47 / 6+49 / 6=16$

$49 / 6+53 / 6=17$

$53 / 6+55 / 6=18$

$55 / 6+59 / 6=19$

$59 / 6+61 / 6=20$

$61 / 6+65 / 6=21$

$65 / 6+67 / 6=22$

$67 / 6+71 / 6=23$

$71 / 6+73 / 6=24$

$73 / 6+77 / 6=25$

$77 / 6+79 / 6=26$

$79 / 6+83 / 6=27$

$83 / 6+89 / 6=28$

$85 / 6+89 / 6=29$

$89 / 6+91 / 6=30$

$91 / 6+95 / 6=31$ 
$95 / 6+97 / 6=32$

$97 / 6+101 / 6=33$

$101 / 6+103 / 6=34$

$103 / 6+107 / 6=35$

$107 / 6+109 / 6=36$

The above, when tallied for the "numerations and the sum total values "yields a stable rhythm of precisely 4,3 , $(3+1 / n), 3,(3+1 / n+2), 3,(3+1 / n+3)$, briefly as follows , simply denoting the stable nonrandom expansion of prime numbers in the matrix of non -linear space. This is as follows. Mathematically the values 2, 3 are not prime numbers. Every alternate value is 3 , and every alternate value is predictable based on the numeration, two cords of this will isolate 3 values (numeration represents the gaps). The values are demonstrated by table following the diagrams.

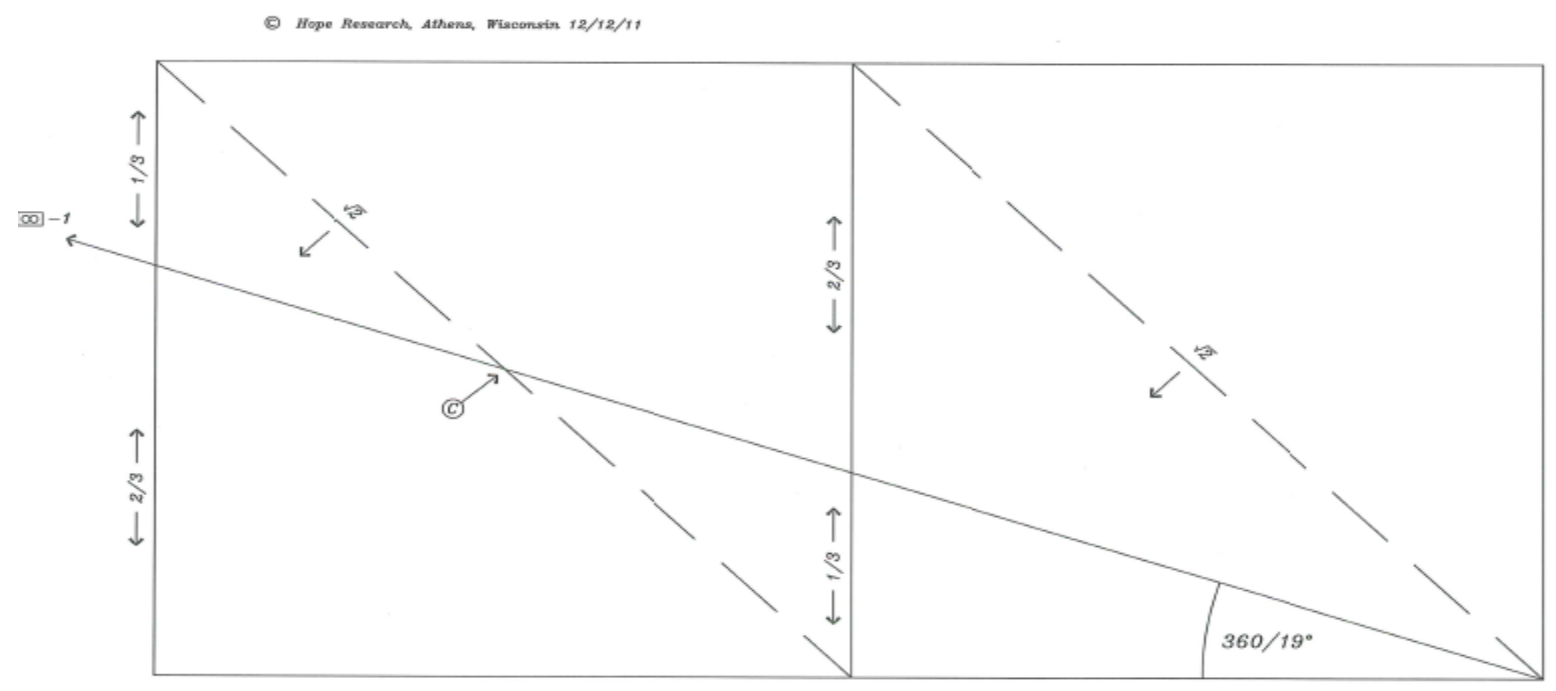

\section{INTRODUCTION}

(C) represents the dead center of the square. An infinite extension of the $360 / 19$ degrees line, infinitely transects all unidimensional squared spaces at $1 / 3,2 / 3$ and (C), in continuum.

$19-\left(\frac{360}{19}\right)=\frac{1}{19} \ldots \ldots \ldots \ldots . . . . . . \frac{\sqrt{2}}{\left(\frac{90}{19}\right)} \cdot 1.11648439134=\frac{1}{3}$
Note: As a rule all calculations are limited to 11 decimal slots

Fig. 1

Representation

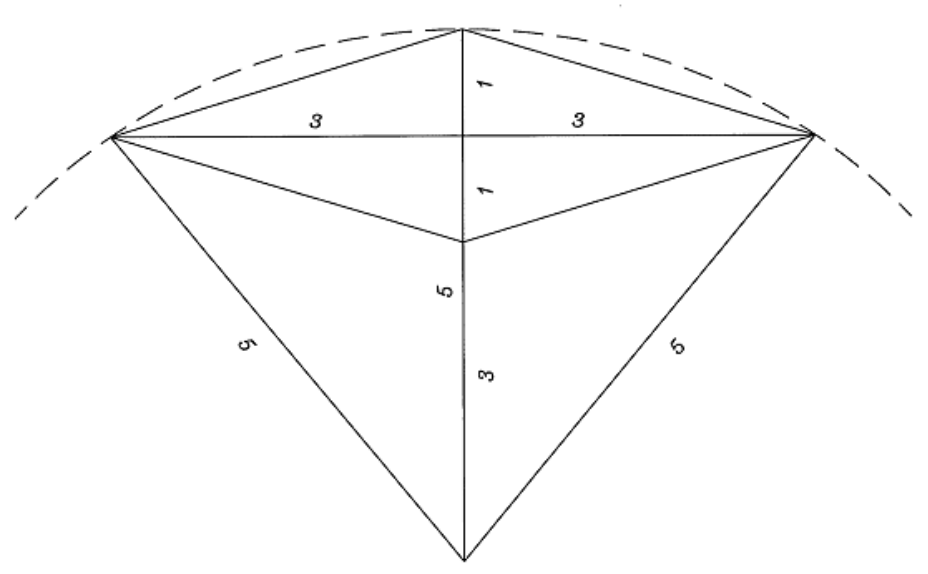

$\frac{5}{6}=\frac{1}{6}-$ Curve constant at -1

Fig. 2 


\begin{tabular}{ccc}
\hline 1 & $(5-1) / 1=$ & 4 \\
2 & $(12-6) / 2=$ & 3 \\
3 & $(23-13) / 3=$ & $3 .+1 / 3$ \\
4 & $(36-24) / 4=$ & 3 \\
5 & $(53-37) / 5=$ & $3 .+1 / 5$ \\
6 & $(72-54) / 6=$ & 3 \\
7 & $(95-73) / 7=$ & $3+1 / 7$ \\
8 & $(120-96) / 8=$ & 3 \\
9 & $(149-121) / 9=$ & $3+1 / 9$ \\
10 & $(180-150) / 10=$ & 3 \\
11 & $(215-181) / 11=$ & $3+1 / 11$ \\
12 & $(252-216) / 12=$ & 3 \\
13 & $(293-253) / 13=$ & $3+1 / 13$ \\
14 & $(336-294) / 14=$ & 3 \\
15 & $(383-337) / 15=$ & $3+1 / 15$ \\
16 & $(432-384) / 16=$ & 3 \\
17 & & \\
18 & & \\
19 & & \\
20 & & \\
\hline
\end{tabular}

\section{Study of the spiral grid of mathematics by a new numbers theorem}

The author has already published a continuous prime number sieve at 1/6 and 5/6 ; and 2/3 and 1/3 (Den-Otter Prime Sieve), but unfortunately in the current obsession with current Mathematics theory, it has not yet been noticed, as current mathematics is celebrating larger and larger prime numbers, to what logic?

The exclusivity of 1:3 for spiral $12(6-\mathrm{x}-6)$ grid has to be noted, should have been noted by the mathematicians, as the grid is equalized by the prime numbers: numbers as shown $(12 / 4=3)$, as an example,

$11+17+23=15+17+19=51$ (prime 17 Spiral 12$)$

$10+16+22=14+16+18=48$ (non- prime $16,1: 3$ spiral 12$)$

(Spiral 12(1:3) grid) and represents the spiral ascension of mathematics at $0.5(2 / 3$ twist and $1 / 3$ twist of the mathematics) is the base grid in which the sum of the regular spirals equals the sum of the prime number spirals. In this concordant grid between the alternate cord of regular numbers with gaps of 4 and the cord of numbers with gaps of 12 are demonstrated for prime numbers. Please note this was discussed in a previous paper that concordance is absolutely equal at a span of 12 and for a span of 16 it is -4 . For a span

There are two constants in this mathematics grid at $1: 3$

A. Constant 3:8, for all numbers in the grid as discussed above in the beginning of the manuscript, prime 17 is an example

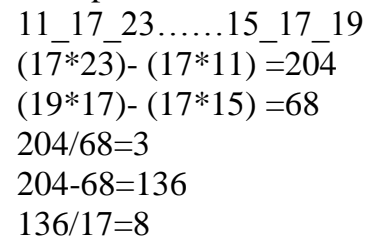

B. Constant 32 , this is from the grid below and is a constant for all numbers

$$
\text { 11_17_23 15_17_19 }
$$

$(19 * 15)-(23 * 11)=32$ Constant for all values in the grid.

The 32 constant leads to the consistent whole number numeration

$(19 * 15) / 32=8.90625$

$(23 * 11) / 32=7.90625$

Starting at the $1^{\text {st }}$ value:

\begin{tabular}{cc}
\hline 1.40625 & 0.40625 \\
1.875 & 0.875 \\
3 & 2 \\
3.65625 & 2.65625 \\
4.375 & 3.375 \\
5.15625 & 4.15625 \\
6 & 5 \\
6.90625 & 5.90625 \\
7.875 & 6.875
\end{tabular}




\begin{tabular}{cc}
8.90625 & 7.90625 \\
10 & 9 \\
11.15625 & 10.15625 \\
12.375 & 11.375 \\
13.65625 & 12.65625 \\
15 & 14 \\
16.40625 & 15.40625 \\
17.875 & 16.875 \\
19.40625 & 18.40625 \\
21 & 20 \\
22.65625 & 21.65625 \\
24.375 & 23.375 \\
28 & 27 \\
29.90625 & 28.90625 \\
31.875 & 30.875 \\
33.90625 & 32.90625 \\
36 & 35 \\
\hline
\end{tabular}

The above translates to a spatial numeration as follows:

$3,6,10,15,21,28,36$, which is,

$(1+2)+(3+3)+(6+4)+(10+5)+(15+6)+(21+7)+(28+8) \ldots \ldots$

$(1 / 2=0.5)+(3 / 3=1)+(6 / 4=1.5)+(10 / 5=2)+(15 / 6=2.5)+(21 / 7=3)+(28 / 8=3.5)$

You can precisely place each number in mathematics in a perfect precise place for that number in the grid. This grid has the following characteristics and is precise and stable. That is how prime numbers are exactly placed:

1) On the right side are three coordinates of regular alternate numbers with a span of 4 , on the left there are three coordinate numbers spirals with a span of 12

2) The sum product of the numbers on the right is precisely equal till infinity to the sum of the numbers on the left $(5+11+17)=(9+11+13)=33$

3) The number 32 is a constant throughout the grid $(13 * 9)-(17 * 5)=32$ for all coordinates

4) Values progress by span count $(3,6,10,15,21,28,36,45,55,66,78,91,105 \ldots)$

5) The grid is predictably concordant with other spiral values i.e12,14,16,18,20... spiral grid values of prime numbers spirals, even though these are available indirectly above, these values by predictive equation seals the final

6) The final equations for the entire spiral Prime number values by direct equation is the EXTENSIVE END CALCULUS EQUATIONS, that which is evident indirectly as the half line spiral values ( span) in the method above and for the first time in history there is predictive Prime number spiral value, when the World is still stuck on Twin Prime numbers. These equations, based on the grids etc. are a no brainer and the author might just keep these secret from the mathematicians, to give the opportunity to mathematicians to recant their mathematics theory

\subsection{Twin prime numbers are predictable and calculable by number series}

This is integral to mathematics that twin Prime numbers are predictable part of each spiral. The following is the twin prime grid: The author has not expended his capital on this as this is integral to the grid of mathematics. These have to be indefinite, and represent the mathematics of the grid, but beyond that this paper is to understand the lay of the forest, and not the natty gritty at this juncture. The prime numbers spirals on the left, the regular number are on the right

Twin Prime numbers placement:

\begin{tabular}{|c|c|c|c|c|c|}
\hline \multicolumn{6}{|c|}{ Spiral10 } \\
\hline 1 & 5 & 11 & 3 & 5 & 7 \\
\hline 3 & 7 & 13 & 5 & 7 & 9 \\
\hline \multicolumn{6}{|c|}{ Spiral12 } \\
\hline 5 & 11 & 17 & 9 & 11 & 13 \\
\hline 7 & 13 & 19 & 11 & 13 & 15 \\
\hline \multicolumn{6}{|c|}{ Spiral14 } \\
\hline \multicolumn{6}{|c|}{ Spiral16 } \\
\hline 31 & 41 & 47 & 39 & 41 & 43 \\
\hline 37 & 43 & 53 & 41 & 43 & 45 \\
\hline \multicolumn{6}{|c|}{ Spiral18 } \\
\hline 17 & 29 & 37 & 27 & 29 & 31 \\
\hline 23 & 31 & 41 & 29 & 31 & 33 \\
\hline 179 & 191 & 197 & 189 & 191 & 193 \\
\hline 181 & 193 & 199 & 191 & 193 & 195 \\
\hline 1279 & 1289 & 1297 & 1287 & 1289 & 1291 \\
\hline 1283 & 1291 & 1301 & 1289 & 1291 & 1293 \\
\hline
\end{tabular}


The Grid of Mathematics At 1:3, Prime Spiral 12 Span

\begin{tabular}{|c|c|c|c|c|c|c|c|c|}
\hline $1(+6)$ & $7(+6)$ & 13 & $5(+2)$ & $7(+2)$ & 9 & +++21 & $=21 / 3$ & 7 \\
\hline 2 & 8 & 14 & 6 & 8 & 10 & +++24 & $=24 / 3$ & 8 \\
\hline 3 & 9 & 15 & 7 & 9 & 11 & +++27 & $=27 / 3$ & 9 \\
\hline 4 & 10 & 16 & 8 & 10 & 12 & +++30 & $=30 / 3$ & 10 \\
\hline $5 *$ & $11 *$ & $17 *$ & $9 *$ & $11 *$ & $13 *$ & +++33 & $=33 / 3$ & 11 \\
\hline 6 & 12 & 18 & 10 & 12 & 14 & +++36 & $=36 / 3$ & 12 \\
\hline $7 *$ & $13 *$ & $19 *$ & $11 *$ & $13 *$ & $15 *$ & +++39 & $=39 / 3$ & 13 \\
\hline 8 & 14 & 20 & 12 & 14 & 16 & +++42 & $=42 / 3$ & 14 \\
\hline 9 & 15 & 21 & 13 & 15 & 17 & +++45 & $=45 / 3$ & 15 \\
\hline 10 & 16 & 22 & 14 & 16 & 18 & +++48 & $=48 / 3$ & 16 \\
\hline $11 *$ & $17 *$ & $23 *$ & $15^{*}$ & $17 *$ & $19 *$ & +++51 & $=51 / 3$ & 17 \\
\hline 12 & 18 & 24 & 16 & 18 & 20 & +++54 & $=54 / 3$ & 18 \\
\hline 13 & 19 & 25 & 17 & 19 & 21 & $(+++57)$ & $=57 / 3$ & 19 \\
\hline 14 & 20 & 26 & 18 & 20 & 22 & +++60 & $=60 / 3$ & 20 \\
\hline 15 & 21 & 27 & 19 & 21 & 23 & +++63 & $=63 / 3$ & 21 \\
\hline 16 & 22 & 28 & 20 & 22 & 24 & +++66 & $=66 / 3$ & 22 \\
\hline 17 & 23 & 29 & 21 & 23 & 25 & +++69 & $=69 / 3$ & 23 \\
\hline 18 & 24 & 30 & 22 & 24 & 26 & +++72 & $=72 / 3$ & 24 \\
\hline 19 & 25 & 31 & 23 & 25 & 27 & +++75 & $=75 / 3$ & 25 \\
\hline 20 & 26 & 32 & 24 & 26 & 28 & +++78 & $=78 / 3$ & 26 \\
\hline 21 & 27 & 33 & 25 & 27 & 29 & +++81 & $=81 / 3$ & 27 \\
\hline 22 & 28 & 34 & 26 & 28 & 30 & +++84 & $=84 / 3$ & 28 \\
\hline 23 & 29 & 35 & 27 & 29 & 31 & +++87 & $=87 / 3$ & 29 \\
\hline 24 & 30 & 36 & 28 & 30 & 32 & +++90 & $=90 / 3$ & 30 \\
\hline 25 & 31 & 37 & 29 & 31 & 33 & +++93 & $=93 / 3$ & 31 \\
\hline 26 & 32 & 38 & 30 & 32 & 34 & +++96 & $=96 / 3$ & 32 \\
\hline 27 & 33 & 39 & 31 & 33 & 35 & +++99 & $=99 / 3$ & 33 \\
\hline 28 & 34 & 40 & 32 & 34 & 36 & +++102 & $=102 / 3$ & 34 \\
\hline 29 & 35 & 41 & 33 & 35 & 37 & +++105 & $=105 / 3$ & 35 \\
\hline 30 & 36 & 42 & 34 & 36 & 38 & +++108 & $=108 / 3$ & 36 \\
\hline 31 & 37 & 43 & 35 & 37 & 39 & +++111 & $=111 / 3$ & 37 \\
\hline 32 & 38 & 44 & 36 & 38 & 40 & $(+++114)$ & $=114 / 3$ & 38 \\
\hline 33 & 39 & 45 & 37 & 39 & 41 & +++117 & $=117 / 3$ & 39 \\
\hline 34 & 40 & 46 & 38 & 40 & 42 & +++120 & $=120 / 3$ & 40 \\
\hline 35 & 41 & 47 & 39 & 41 & 43 & +++123 & $=123 / 3$ & 41 \\
\hline 36 & 42 & 48 & 40 & 42 & 44 & +++126 & $=126 / 3$ & 42 \\
\hline 37 & 43 & 49 & 41 & 43 & 45 & +++129 & $=129 / 3$ & 43 \\
\hline 38 & 44 & 50 & 42 & 44 & 46 & +++132 & $=132 / 3$ & 44 \\
\hline 39 & 45 & 51 & 43 & 45 & 47 & +++135 & $=135 / 3$ & 45 \\
\hline 40 & 46 & 52 & 44 & 46 & 48 & +++138 & $=138 / 3$ & 46 \\
\hline 41 & 47 & 53 & 45 & 47 & 49 & +++141 & $=148 / 3$ & 47 \\
\hline 42 & 48 & 54 & 46 & 48 & 50 & +++144 & $=144 / 3$ & 48 \\
\hline 43 & 49 & 55 & 47 & 49 & 51 & +++147 & $=147 / 3$ & 49 \\
\hline 44 & 50 & 56 & 48 & 50 & 52 & +++150 & $=150 / 3$ & 50 \\
\hline 45 & 51 & 57 & 49 & 51 & 53 & +++153 & $=153 / 3$ & 51 \\
\hline 46 & 52 & 58 & 50 & 52 & 54 & +++156 & $=156 / 3$ & 52 \\
\hline 47 & 53 & 59 & 51 & 53 & 55 & +++159 & $=159 / 3$ & 53 \\
\hline 48 & 54 & 60 & 52 & 54 & 56 & +++162 & $=162 / 3$ & 54 \\
\hline 49 & 55 & 61 & 53 & 55 & 57 & +++165 & $=165 / 3$ & 55 \\
\hline 50 & 56 & 62 & 54 & 56 & 58 & +++168 & $=168 / 3$ & 56 \\
\hline 51 & 57 & 63 & 55 & 57 & 59 & $(+++171)$ & $=171 / 3$ & 57 \\
\hline 52 & 58 & 64 & 56 & 58 & 60 & +++174 & $=174 / 3$ & 58 \\
\hline 53 & 59 & 65 & 57 & 59 & 61 & +++177 & $=177 / 3$ & 59 \\
\hline 54 & 60 & 66 & 58 & 60 & 62 & +++180 & $=180 / 3$ & 60 \\
\hline 55 & 61 & 67 & 59 & 61 & 63 & +++183 & $=183 / 3$ & 61 \\
\hline 56 & 62 & 68 & 60 & 62 & 64 & +++186 & $=186 / 3$ & 62 \\
\hline 57 & 63 & 69 & 61 & 63 & 65 & +++189 & $=189 / 3$ & 63 \\
\hline 58 & 64 & 70 & 62 & 64 & 66 & +++192 & $=192 / 3$ & 64 \\
\hline
\end{tabular}




\begin{tabular}{|c|c|c|c|c|c|c|c|c|}
\hline 59 & 65 & 71 & 63 & 65 & 67 & +++195 & $=195 / 3$ & 65 \\
\hline 60 & 66 & 72 & 64 & 66 & 68 & +++198 & $=198 / 3$ & 66 \\
\hline 61 & 67 & 73 & 65 & 67 & 69 & +++201 & $=201 / 3$ & 67 \\
\hline 62 & 68 & 74 & 66 & 68 & 70 & +++204 & $=204 / 3$ & 68 \\
\hline 63 & 69 & 75 & 67 & 69 & 71 & +++207 & $=207 / 3$ & 69 \\
\hline 64 & 70 & 76 & 68 & 70 & 72 & +++210 & $=210 / 3$ & 70 \\
\hline 65 & 71 & 77 & 69 & 71 & 73 & +++213 & $=213 / 3$ & 71 \\
\hline 66 & 72 & 78 & 70 & 72 & 74 & +++216 & $=216 / 3$ & 72 \\
\hline 67 & 73 & 79 & 71 & 73 & 75 & +++219 & $=219 / 3$ & 73 \\
\hline 68 & 74 & 80 & 72 & 74 & 76 & +++222 & $=222 / 3$ & 74 \\
\hline 69 & 75 & 81 & 73 & 75 & 77 & +++225 & $=225 / 3$ & 75 \\
\hline 70 & 76 & 82 & 74 & 76 & 78 & $(+++228)$ & $=228 / 3$ & 76 \\
\hline 71 & 77 & 83 & 75 & 77 & 79 & +++231 & $=231 / 3$ & 77 \\
\hline 72 & 78 & 84 & 76 & 78 & 80 & +++234 & $=234 / 3$ & 78 \\
\hline 73 & 79 & 85 & 77 & 79 & 81 & +++237 & $=237 / 3$ & 79 \\
\hline 74 & 80 & 86 & 78 & 80 & 82 & +++240 & $=240 / 3$ & 80 \\
\hline 75 & 81 & 87 & 79 & 81 & 83 & +++243 & $=243 / 3$ & 81 \\
\hline 76 & 82 & 88 & 80 & 82 & 84 & +++246 & $=246 / 3$ & 82 \\
\hline 77 & 83 & 89 & 81 & 83 & 85 & +++249 & $=249 / 3$ & 83 \\
\hline 78 & 84 & 90 & 82 & 84 & 86 & +++252 & $=252 / 3$ & 84 \\
\hline 79 & 85 & 91 & 83 & 85 & 87 & +++255 & $=255 / 3$ & 85 \\
\hline 80 & 86 & 92 & 84 & 86 & 88 & +++258 & $=258 / 3$ & 86 \\
\hline 81 & 87 & 93 & 85 & 87 & 89 & +++261 & $=261 / 3$ & 87 \\
\hline 82 & 88 & 94 & 86 & 88 & 90 & +++264 & $=264 / 3$ & 88 \\
\hline 83 & 89 & 95 & 87 & 89 & 91 & +++267 & $=267 / 3$ & 89 \\
\hline 84 & 90 & 96 & 88 & 90 & 92 & +++270 & $=270 / 3$ & 90 \\
\hline 85 & 91 & 97 & 89 & 91 & 93 & +++273 & $=273 / 3$ & 91 \\
\hline 86 & 92 & 98 & 90 & 92 & 94 & +++276 & $=276 / 3$ & 92 \\
\hline 87 & 93 & 99 & 91 & 93 & 95 & +++279 & $=279 / 3$ & 93 \\
\hline 88 & 94 & 100 & 92 & 94 & 96 & +++282 & $=282 / 3$ & 94 \\
\hline 89 & 95 & 101 & 93 & 95 & 97 & $(+++285)$ & $=285 / 3$ & 95 \\
\hline 90 & 96 & 102 & 94 & 96 & 98 & +++288 & $=288 / 3$ & 96 \\
\hline 91 & 97 & 103 & 95 & 97 & 99 & +++291 & $=291 / 3$ & 97 \\
\hline 92 & 98 & 104 & 96 & 98 & 100 & +++294 & $=294 / 3$ & 98 \\
\hline 93 & 99 & 105 & 97 & 99 & 101 & +++297 & $=297 / 3$ & 99 \\
\hline 94 & 100 & 106 & 98 & 100 & 102 & +++300 & $=300 / 3$ & 100 \\
\hline 95 & 101 & 107 & 99 & 101 & 103 & +++303 & $=303 / 3$ & 101 \\
\hline 96 & 102 & 108 & 100 & 102 & 104 & +++306 & $=306 / 3$ & 102 \\
\hline $97 *$ & $103 *$ & $109 *$ & $101^{*}$ & $103 *$ & $105^{*}$ & +++309 & $=309 / 3$ & 103 \\
\hline 98 & 104 & 110 & 102 & 104 & 106 & +++312 & $=312 / 3$ & 104 \\
\hline 99 & 105 & 111 & 103 & 105 & 107 & +++315 & $=315 / 3$ & 105 \\
\hline 100 & 106 & 112 & 104 & 106 & 108 & +++318 & $=318 / 3$ & 106 \\
\hline $101 *$ & $107^{*}$ & $113^{*}$ & $105^{*}$ & $107 *$ & $109 *$ & +++321 & $=321 / 3$ & 107 \\
\hline 1481* & $1487 *$ & 1493* & $1485 *$ & $1487 *$ & 1491 & +++4459 & $=4459 / 3$ & 1487 \\
\hline
\end{tabular}

\section{Conclusion}

This is clearly and singularly the solution to prime number spiral calculus and the absolute arrangement of the entire mathematics, which is reasonably simple after this discovery. The ruminations of George Riemann and others in linear ascension mathematics of prime numbers are non-functional irrelevant mathematics, just collateral observations of the correct mathematics, as was the observations of Einstein. In Mathematics there is only a single best resolution, whilst collateral equations abound, as they have for 500 years for prime numbers which we have proved are basically by two cords and ascend by a half-line and concordance with regular number.

Alternate Regular numbers at base of 4 are totally and precisely concordant at with all the numbers with the base span of 12, and including all Prime number spirals with a base span offset at 12 . 


\section{Acknowledgements}

1) Jesus Christ for his grace and the inspiration of the blessed Virgin Mary first and foremost in my case (And due respect for all the good in all religions, including Islam, Buddhism, Hinduism, Judaism, Sikhism) who gave the author humility, wisdom, inspiration and standing amongst all the so called super mathematicians, by his special grace, truth, to throw the houlihan of mathematics. (A cowboy's lasso).

2) Dr. Belal Mohammed Batiha, $\mathrm{PhD}$, a magnanimous humble mathematician and human being to the author, Editor of IJAMR

3) Dr. Hong Ma, PhD, the brave editor of Journal of American science, for his pragmatism that the best ideas come from the least expected sources

4) Janice Kay my long suffering wife Janice Kay, a thank you for simple mathematical insights.

5) IJAMR, for its integrity and open mindedness; unlike many other journals in mathematics, they do not pinch the nostrils of creativity, neither do they direct the milk of liberty in the sciences into their own suckers. Knowledge is for the free, not the elite.

\section{References}

[1] Cameron.V. The first ever precise predictive prime number placement, International journal of applied mathematics research 2(3) (2013)345351) (journal listed by the American mathematics) society)

[2] Cameron. V. The spiral code of prime numbers, International journal of applied Mathematics research 2(2) (2013)279-292)

[3] Cameron. V. The unified Theorem at -1 (Vedic Zero), International Journal of Mathematics research, 2(2) (2013) 221-251)

[4] Cameron .V, The disproof and fall of the Riemann's hypothesis by quadratic base: The correct variable distribution of prime numbers by the clear mathematics of the half-line values ("Chan function") of prime numbers, International Journal of Applied Mathematical Research, 2 (1) (2013) 103-110.

[5] Cameron V, den Otter T. Prime numbers 2012. Jam Sci 2012; 8(7):329-334]. (ISSN: 1545-1003), http://www.jofamericanscience.org.

[6] Cameron V, Prime number Coordinates and calculus J Am Sci, 2012; 8(10):9-10]. (ISSN: 1545-1003).http://www.jofamericanscience.org

[7] Cameron: Prime number19, Vedic Zero and the fall of western mathematics by theorem. International journal of applied mathematical research 2(1) (2013)111-115

[8] Cameron: The rational variability of all empty space by prime number: International journal of applied mathematical research, 2(2) (2013)157174

[9] Cameron :The poison pill of current mathematics theory, delivered : International journal of applied mathematical research;2(3)(2013)387402) 